\title{
Globe
}

\section{Le croisement des savoirs et des pratiques avec des personnes en situation de pauvreté. Une condition nécessaire pour une société du savoir inclusive Merging Knowledge and Practices with People Experiencing Poverty. An Essential Condition for an Inclusive Knowledge Society}

\author{
Marianne de Laat, Sophie Boyer, Catherine Hudon, Émilie Goulet et Christine \\ Loignon
}

Volume 17, numéro 2, 2014

Les enjeux du modèle des sociétés du savoir tels qu'ils sont vécus au Québec

URI : https://id.erudit.org/iderudit/1036238ar

DOI : https://doi.org/10.7202/1036238ar

Aller au sommaire du numéro

Éditeur(s)

Globe, Revue internationale d'études québécoises

ISSN

1481-5869 (imprimé)

1923-8231 (numérique)

Découvrir la revue

Citer cet article

de Laat, M., Boyer, S., Hudon, C., Goulet, É. \& Loignon, C. (2014). Le croisement des savoirs et des pratiques avec des personnes en situation de pauvreté. Une condition nécessaire pour une société du savoir inclusive. Globe, 17(2), 69-91. https://doi.org/10.7202/1036238ar

\section{Résumé de l'article}

La démarche de croisement des savoirs et des pratiques entre personnes en situation de pauvreté, universitaires et praticiens repose sur une conviction à la fois éthique et épistémologique : toute personne détient potentiellement les moyens de comprendre et d'interpréter sa propre situation. C'est l'analyse du vécu des personnes en situation de pauvreté et d'exclusion qui est le point de départ d'une construction de savoirs croisés. Il s'agit en fait d'une fécondation réciproque, chacun des partenaires de l'échange apportant à l'autre les éléments d'une production et d'une transformation de son propre savoir. Il ne s'agit donc pas de plaider pour un savoir unique et uniforme, sorte de synthèse des différents types de savoirs. Chacun existe comme acteur et auteur à part entière de sa propre pensée et de son action, comme détenteur d'un savoir reconnu par les autres acteurs. Par le croisement des savoirs et des pratiques, l'effort de construction par chacun de son propre savoir et la coconstruction d'un produit commun sont associés. Il s'agit de déconstruire pour reconstruire, de se laisser interpeller et d'accepter le déplacement de son point de vue. Au Québec, la recherche ÉQUIsanTÉ, une recherche participative menée au sein de deux unités de médecine de famille de l'Université de Sherbrooke, s'appuie sur la démarche du croisement des savoirs : les personnes en situation de pauvreté, les cliniciens et cliniciennes et les universitaires ont agi à titre de cochercheurs du début (formulation de la question de recherche) jusqu'à la fin de la recherche (écriture collective et diffusion des résultats). 


\title{
LE CROISEMENT \\ DES SAVOIRS ET DES \\ PRATIQUES AVEC DES \\ PERSONNES EN SITUATION \\ DE PAUVRETÉ. UNE \\ CONDITION NÉCESSAIRE \\ POUR UNE SOCIÉTÉ \\ DU SAVOIR INCLUSIVE
}

\author{
MARIANNE DE LAAT \\ Mouvement ATD Quart Monde (France) \\ SOPHIE BOYER \\ Mouvement ATD Quart Monde (Canada)
}

\section{CATHERINE HUDON, ÉMILIE GOULET, CHRISTINE LOIGNON \\ Université de Sherbrooke (Canada)}

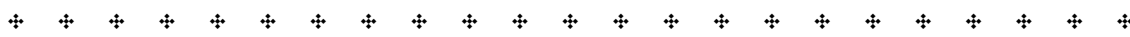

Résumé - La démarche de croisement des savoirs et des pratiques entre personnes en situation de pauvreté, universitaires et praticiens repose sur une conviction à la fois éthique et épistémologique : toute personne détient potentiellement les moyens de comprendre et d'interpréter sa propre situation. C'est l'analyse du vécu des personnes en situation de pauvreté et d'exclusion qui est le point de départ d'une construction de savoirs croisés. Il s'agit en fait d'une fécondation réciproque, chacun des partenaires de l'échange apportant à l'autre les éléments d'une production et d'une transformation de son propre savoir. Il ne s'agit donc pas de plaider pour un savoir unique et uniforme, sorte de synthèse des différents types de savoirs. Chacun existe comme acteur et auteur à part entière de sa propre pensée et de son action, comme détenteur d'un savoir reconnu par les autres acteurs. Par le croisement des savoirs et des pratiques, l'effort de construction par chacun de son propre savoir et la coconstruction d'un produit commun sont associés. Il s'agit de déconstruire pour reconstruire, de se laisser interpeller et d'accepter le déplacement de son point de vue. Au Québec, la recherche ÉQUIsanTÉ, une recherche participative menée au sein de deux unités de médecine de famille de l'Université de Sherbrooke, s'appuie sur la démarche du croisement des savoirs : les personnes en situation 
de pauvreté, les cliniciens et cliniciennes et les universitaires ont agi à titre de cochercheurs du début (formulation de la question de recherche) jusqu’à la fin de la recherche (écriture collective et diffusion des résultats).

\title{
Merging Knowledge and Practices with People Experiencing Poverty. An Essential Condition for an Inclusive Knowledge Society
}

\begin{abstract}
Involving people living in poverty, academics, and practitioners in the process of Merging Knowledge and Practices requires a belief that is both ethical and epistemological: that every individual potentially has the means to understand and interpret his or her own circumstances. The starting point for building merged knowledge is an analysis of the lived experiences of people living with poverty and exclusion. It is a process of cross-fertilization that involves all participants in the process and that allows them to share the elements necessary to produce and transform their own knowledge. It is not a matter of advocating for singular or uniform knowledge, or for some kind of synthesis of different types of knowledge. Each individual participates both as an actor and as the author of his or her own thoughts and actions, possessing a knowledge that is recognized by other participants. The Merging of Knowledge and Practices involves uniting each person's efforts to build his or her own knowledge with a collective effort to build a common product. It is a matter of deconstructing in order to rebuild, of opening one's mind to others' ideas, and of seeing things from another point of view. In Quebec, EQUIhealThY - a participatory research project focused on merging the knowledge of people living in poverty, academics, and practitioners - was undertaken in two Family Medicine Units at the University of Sherbrooke. All participants assumed the role of co-researchers, from start (formulation of research questions) to finish (collective writing and publication of results).
\end{abstract}

$+4+4+4+4+4+4+4+4+4+4$

L'UNESCO, dans son rapport mondial Vers les sociétés du savoir, nous propose un idéal: «Les sociétés du savoir ne mériteront vraiment leur nom que si le plus grand nombre possible d'individus peuvent devenir producteurs de savoirs et ne demeurent pas simplement consommateurs du savoir actuellement disponible ${ }^{1}$ ». Ou encore: «Les sociétés du savoir du XXI ${ }^{\mathrm{e}}$ siècle ne pourront déboucher sur une nouvelle ère de développement humain et durable qu'à condition d'assurer non seulement un accès universel au savoir, mais encore une participation de tous aux sociétés de la connaissance ${ }^{2}$ ».

Le Mouvement ATD Quart Monde, créé en 1957 par Joseph Wresinski et des familles en grande pauvreté du camp des sans-logis de

$$
4+4
$$

1. Jérôme BINDÉ, Vers les sociétés du savoir. Rapport mondial de l'UNESCO, Paris, Éditions UNESCO, 2005, p. 99.

2. Ibid., p. 169.

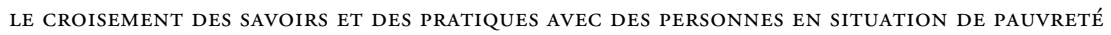


Noisy-le-Grand (France), est un mouvement international de lutte contre la pauvreté. Son principal objectif est l'accès de tous aux droits de tous, par la mobilisation de tous. Par leurs expérimentations, leurs recherches et leurs formations, les membres du mouvement n'ont cessé, depuis son origine, de s'interroger sur les connaissances dont ont besoin les personnes les plus pauvres et exclues, sur les connaissances dont ont besoin les équipes d'action et sur les connaissances dont ont besoin nos sociétés nationales et les communautés internationales pour combattre efficacement la pauvreté et l'exclusion ${ }^{3}$.

En 1980, Joseph Wresinski s'adressait ainsi aux chercheurs :

Ce dont je voulais vous parler ce matin [...] c'est de la fonction (et je dirais volontiers du devoir) des chercheurs dans le domaine de la pauvreté, de faire place à la connaissance que les très pauvres euxmêmes ont de leur condition. De faire place à cette connaissance, de la réhabiliter comme unique et indispensable, autonome et complémentaire à toute autre forme de connaissance, et de l'aider à se développer ${ }^{4}$.

Cet appel aux chercheurs et chercheuses universitaires repose sur la conviction que les personnes en situation de grande pauvreté et d'exclusion savent mieux que d'autres ce que devrait être une société juste et fraternelle et jusqu'où devraient aller les efforts d'une société pour que les droits de la personne soient effectifs pour tous, pour que personne ne soit oublié dans le développement humain et durable. Mais si les institutions responsables du savoir ne reconnaissent pas le savoir et la capacité contributive des personnes en situation de pauvreté et d'exclusion, comment ces personnes pourraientelles y croire elles-mêmes? Comment parviendraient-elles à se faire entendre d'une société qui ne voit souvent en elles que des manques et des besoins? Est-il possible que des universitaires s'intéressent au point de vue des personnes en situation de pauvreté et d'exclusion et prennent les moyens de les rencontrer, de les comprendre et de s'associer avec elles pour faire reconnaître publiquement la valeur de leurs savoirs?

Celui ou celle qui échoue à l'école, qui vit de l'aide de dernier recours depuis trop longtemps, qui n'a pas de logement, qui ne peut se soigner a quelque chose d'essentiel à partager sur le respect des droits de la

$$
\div+\div
$$

3. Claude FERrAND, «De l'exclusion sociale et culturelle à la pédagogie du croisement des savoirs », Pensée plurielle, $\mathrm{n}^{\circ}$ 3, 2001.

4. Joseph WRESINSKI, "La pensée des plus pauvres dans une connaissance qui conduise au combat", Refuser la misère - Une pensée politique née de l'action, Paris, Éditions Cerf et Quart Monde, 2007, p. 52. 
personne. Si l'analyse de ce vécu et de ces expériences n'est pas prise en compte, les politiques publiques qui visent à lutter contre la pauvreté et à assurer un développement humain et durable pour tous n'atteindront pas les citoyens et citoyennes en situation de grande pauvreté. Le discours dominant continuera de les culpabiliser, les accusant de ne pas vouloir faire des efforts pour utiliser les dispositifs mis en place pour les aider.

Heureusement, la participation des personnes en situation de pauvreté et d'exclusion à la production des savoirs, même si elle est encore rare, n'est plus une utopie. Des chercheurs et chercheuses abattent le mur dressé entre les producteurs officiels de savoirs (les scientifiques) et les autres et considèrent les personnes en grandes difficultés économiques, sociales et culturelles comme des partenaires à part entière, capables de formuler leurs avis, leurs réflexions et leurs analyses, permettant ainsi la production de savoirs qui apportent des réponses plus justes et efficaces.

Dans cet article, nous développerons ce qu'est la démarche du croisement des savoirs et des pratiques entre des personnes en situation de pauvreté, des universitaires et des praticiens et praticiennes en faisant référence à son origine, deux recherches-action-formation franco-belges menées par le Mouvement ATD Quart Monde, et en nous appuyant sur la recherche participative ÉQUIsanTÉ, qui s'est déroulée au Québec dans le domaine de la santé.

\section{LE CROISEMENT DES SAVOIRS ET DES PRATIQUES : SES FONDEMENTS ET SES ENJEUX}

La démarche de croisement des savoirs et des pratiques entre personnes en situation de pauvreté, universitaires et praticiens et praticiennes repose sur une position à la fois éthique et épistémologique : toute personne détient potentiellement les moyens de comprendre et d'interpréter sa propre situation. Comme l'exprime Jacques Rancière: "Il s'agissait de poser qu'intellectuel, tout le monde l'est, au sens où chacun use de sa tête, au sens aussi où il n'y a pas différentes manières d'user de sa tête... ". C'est «l'affirmation de l'égale intelligence, de l'égale capacité de n'importe qui, de tout collectif de manifestation et d'énonciation, à formuler les termes d'une question politique $^{6} »$. C'est l'analyse du vécu, le savoir expérientiel des personnes en

$$
4+4
$$

5. Mathieu POTTE-BONNEVILlE et Isabelle SAINT-SAËNS, "Le maitre ignorant, entretien avec Jacques Rancière", Vacarme, no 3, 1999, p. 4-8.

6. Ibid., p. 7. 
situation de pauvreté et d'exclusion, qui est le point de départ d'une construction de savoirs croisés.

Le croisement des savoirs est une fécondation réciproque dans laquelle chacun des partenaires de l'échange apporte à l'autre les éléments d'une production et d'une transformation de son propre savoir. Il n'est donc pas question, pour cette démarche, de plaider pour un savoir unique et uniforme, qui serait une sorte de synthèse des différents types de savoirs. Chaque personne existe et est reconnue par les autres comme actrice et auteure à part entière de sa propre pensée et de son action, comme détentrice d'un savoir pertinent et intéressant. Le croisement des savoirs et des pratiques consiste à associer l'effort de construction par chaque personne de son propre savoir avec la coconstruction d'un savoir commun. L'objectif d'un croisement est de déconstruire pour reconstruire, de se laisser interpeller et d'accepter le déplacement de son point de vue.

Ce croisement des savoirs va à l'encontre des rapports inégalitaires que l'ordre social impose aux savoirs de personnes au statut social différencié. Un enjeu de la démarche du croisement des savoirs est donc de réhabiliter, au bénéfice de tous, la contribution intellectuelle et pratique issue de l'analyse du vécu, de l'expérience des personnes en situation de grande pauvreté et d'exclusion. Ce savoir est bien là, même s'il n'est pas socialement reconnu comme tel et même s'il est empêché de s'exprimer et de se construire à cause de la misère et de la stigmatisation des personnes qui la vivent. Lui donner les moyens d'émerger, de se reconnaître et de se confronter aux autres savoirs est, dans cette perspective, une exigence démocratique et une nécessité pour lutter efficacement contre la grande pauvreté et l'exclusion sociale. Cette reconnaissance entraîne l'empowerment, le développement du pouvoir d'agir de personnes qui reprennent ainsi confiance en elles et en leurs moyens.

Mais comment faire? Un autre enjeu de la démarche du croisement des savoirs est de créer les conditions de rencontre et de dialogue entre trois sources de savoirs, représentées par différents acteurs: le savoir universitaire ou théorique, le savoir existentiel et expérientiel des personnes qui vivent des conditions de grande pauvreté et qui luttent pour s'en sortir et le savoir d'action et d'engagement. Ces trois types de savoirs s'ignorent la plupart du temps et entrent parfois en conflit, ce qui crée des malentendus, des incompréhensions et des exclusions. D'où la nécessité de créer les conditions nécessaires pour que ces savoirs se rencontrent, s'enrichissent et se corrigent afin d'éliminer la misère et l'exclusion. 


\section{DEUX PROGRAMMES PILOTES FRANCO-BELGES}

C'est par la réalisation de deux programmes pilotes franco-belges qu'ATD Quart Monde a expérimenté les conditions du croisement des savoirs et des pratiques: Quart Monde Université (1996-1998) et Quart Monde Partenaire (1999-2001). Des personnes issues de la grande pauvreté et membres des universités populaires Quart Monde ${ }^{7}$, des universitaires et des praticiens et praticiennes ont mené ces deux recherches-action-formation.

La première recherche, Quart Monde Université ${ }^{8}$, est partie du constat que la misère est un déni des droits de la personne et que les populations en situation de pauvreté et d'exclusion sociale ne contribuent pas à la connaissance. Pourtant, leur savoir expérientiel est indispensable pour comprendre et analyser ce qu'elles vivent et comment fonctionne la société et pour savoir ce qu'il faut pour la changer, à partir de leur histoire, de leurs conditions de vie et de leurs luttes quotidiennes. Les personnes en situation de pauvreté sont bien souvent l'objet de recherches sans en être les coactrices ou sans être reconnues comme cochercheuses. La recherche et l'enseignement se privent ainsi d'une connaissance précieuse.

Trente-deux personnes ont participé à la recherche Quart Monde Université, dont quinze ayant l'expérience de la grande pauvreté, douze universitaires et cinq volontaires permanents ${ }^{9}$ d'ATD Quart Monde. Les personnes qui ont l'expérience de la pauvreté avaient toutes participé depuis plusieurs années aux universités populaires Quart Monde, où elles ont acquis des capacités d'écoute et d'expression. Elles représentaient le savoir expérientiel. Les universitaires provenaient de dix universités et de huit disciplines différentes: le droit, l'économie, les sciences de l'éducation, la médecine, la physique, la criminologie, l'histoire et la sociologie. Ils représentaient le savoir universitaire. Les volontaires permanents d'ATD Quart Monde avaient des origines culturelles et professionnelles différentes et exerçaient des fonctions variées au sein d'ATD Quart Monde. Ils représentaient le savoir de l'action.

$$
\div \div
$$

7. Université populaire Quart Monde : lieu de rencontre, de formation réciproque entre des adultes vivant la pauvreté et des citoyens qui s'engagent à leurs côtés.

8. Groupe De ReCherChe QuART Monde Université, Le Croisement des savoirs. Quand le Quart Monde et l'université pensent ensemble, Paris, Atelier/éditions ouvrières Quart Monde, 1999.

9. Les volontaires permanents du Mouvement ATD Quart Monde sont des hommes et des femmes qui choisissent de s'engager à plein temps et dans la durée aux côtés des personnes en grande pauvreté. Ils sont près de 400 dans le monde, originaires d'une quarantaine de pays, de convictions philosophiques et religieuses distinctes et de formations et de métiers différents. Ils reçoivent une indemnité modeste qui est la même pour tous (http://www.atd-quartmonde.org/-Rejoignez-le-Volontariat-Permanent-.html). 
Dans la recherche, trois étapes peuvent être distinguées : la construction des questions de recherche, l'analyse des données et l'écriture des résultats. Lors du premier séminaire qui regroupait les trente-deux participants et participantes, chacun a réalisé son blason ${ }^{10}$ (composé d'une devise, des questions qu'on voulait porter dans la recherche, des expériences fondatrices de valeurs, d'une image ou d'un symbole) et l'a présenté aux autres. Au-delà de la reconnaissance interpersonnelle, cette rencontre autour des blasons a permis de capitaliser l'état des questions et des réflexions portées par les différentes personnes. C'est à partir de ce recueil et de cette mise en relation des expériences et des savoirs à propos de la misère que le questionnement collectif s'est ensuite élaboré. Ce premier séminaire a été le lancement de la recherche, qui a duré deux ans.

Au cours de la recherche, un travail de base a été fourni par les personnes en situation de pauvreté, qui travaillaient trois jours par semaine en sous-groupes de trois. Chaque sous-groupe était soutenu, une demijournée par semaine, par une personne-ressource, enseignante de formation. Un travail collectif du groupe des trente-deux personnes a été effectué sous la forme de séminaires de trois jours tous les deux mois, soit dix séminaires. Des groupes thématiques composés par des personnes en situation de pauvreté, des universitaires et des volontaires permanents travaillaient ensemble sur un thème précis et assuraient le travail de production des connaissances.

Cinq thèmes de recherche ont été élaborés par les participants et participantes : le savoir et l'école; le projet familial et le temps; l'histoire du passage de la honte à la fierté; le travail et les savoir-faire des très pauvres; la citoyenneté et la représentation. Les participants et participantes ont par la suite élaboré cinq problématiques, qui, chacune, ont fait l'objet d'un mémoire de recherche coécrit.

La deuxième recherche, Quart Monde Partenaire ${ }^{11}$, partait du constat qu'il existe des malentendus et des incompréhensions réciproques entre, d'un côté, les personnes et les populations en situation de grande pauvreté et, de l'autre, les institutions et les professionnels chargés de leur venir en aide ou de répondre à leurs demandes. L'objectif de ce programme était de produire une méthode de rencontre et de dialogue à travers une

$$
4 * *
$$

10. Pascal Galvani, Quête de sens et formation. Anthropologie du blason et de l'autoformation, Paris, L'Harmattan, 1997.

11. GROUPE DE RECHERCHE ACTION-FORMATION QUART MONDE PARTENAIRE, Le croisement des pratiques. Quand le Quart Monde et les professionnels se forment ensemble, Paris, Éditions Quart Monde, 2002 . 
expérience de coformation à la connaissance réciproque et au partenariat avec les personnes en situation de pauvreté pour améliorer les interactions entre les différents acteurs.

Trente-six personnes ont participé à cette recherche : seize praticiens et praticiennes dans différents domaines (enseignement, santé, travail social, habitat, petite enfance, justice, culture...) et vingt-deux membres du Mouvement ATD Quart Monde, dont seize issus d'une situation de grande pauvreté ou vivant alors une telle situation, trois alliés, c'est-à-dire des personnes de tous les milieux qui soutiennent la lutte contre la pauvreté, et deux volontaires permanents.

Plutôt que de rassembler des réflexions de manière abstraite, l'équipe pédagogique a préféré une démarche de type expérientiel: chaque personne a d'abord écrit un récit d'expérience qui relatait les interactions vécues entre des personnes vivant dans la pauvreté et des praticiens et praticiennes mandatés par leurs institutions. Il a ensuite été demandé que chaque personne lise les récits des autres membres de son groupe de pairs en repérant, dans chaque texte, les passages où se produisait quelque chose d'important, de positif ou de négatif, qui orientait l'évolution de l'action et des relations entre les personnes. C'est la mise en commun de la lecture et de l'analyse des récits qui a permis d'identifier un certain nombre de nœuds, qui ont ensuite donné lieu à cinq thématiques de travail : logiques institutionnelles et logiques de la personne; connaissance et représentations; nature de la relation; participation et conditions pour être acteurs; et initiatives et prises de risque.

Chaque groupe thématique a enfin relu tous les récits en s'efforçant de rassembler des matériaux autour de sa propre problématique. C'est l'analyse des récits qui a permis de trouver des conditions nécessaires pour améliorer les interactions entre personnes en difficulté et praticiens et praticiennes $^{12}$.

Pendant les cinq ans qu'ont duré ces deux recherches, une équipe pédagogique a créé des conditions nécessaires au bon fonctionnement et inventé pas à pas une pédagogie, une méthode et une éthique du croisement des savoirs et des pratiques. Cette équipe était composée de :

- un directeur du programme, volontaire permanent du Mouvement ATD Quart Monde ;

$$
\div+\div
$$

12. Pour obtenir plus de détails sur la méthodologie de cette recherche, voir ibid., p. 27-35. 
- deux universitaires, conseillers à la formation, chargés de conseiller les universitaires, les volontaires permanents et les praticiens et praticiennes;

- une conseillère pédagogique, volontaire permanente, chargée d'accompagner les personnes en situation de pauvreté et d'être la personne ressource en matière de pédagogie pour l'ensemble du programme;

- et un universitaire évaluateur, chargé de capitaliser la connaissance issue de la recherche au fur et à mesure de sa production et d'en évaluer les différents aspects.

Dans une démarche visant à faire reconnaître le croisement de savoirs comme une source valide de connaissance par l'institution universitaire, un comité scientifique composé d'universitaires et un conseil d'orientation regroupant des représentants et représentantes des ministères et des institutions ayant mandaté des praticiens et praticiennes ont validé, sur la base d'une évaluation, les produits de cette expérience, à savoir une méthode de corecherche entre les universitaires et les personnes en situation de pauvreté sur des sujets de société et une méthode de coformation entre les praticiens et praticiennes agissant dans différents domaines et les personnes en situation de pauvreté. Depuis, la démarche du croisement des savoirs se développe et s'adapte en fonction de la charte du croisement des savoirs et des pratiques ${ }^{13}$, écrite par un groupe de personnes ayant participé à ces deux programmes de recherche. Cette charte reprend les prérequis et les conditions nécessaires pour la mise en œuvre d'une démarche de croisement des savoirs. Nous y reviendrons plus loin dans l'article.

\section{ÉQUIsanTÉ, UNE RECHERCHE PARTICIPATIVE QUÉBÉCOISE S'APPUYANT SUR LA DÉMARCHE DU CROISEMENT DES SAVOIRS}

En s'appuyant, d'une part, sur la charte du croisement des savoirs et des pratiques mise en œuvre par le Mouvement ATD Quart Monde et, d'autre part, sur une démarche d'application des connaissances intégrée $(\mathrm{ACI})^{14}$,

$$
4+4
$$

13. Mouvement ATD QuART MONDE - ATELIERS DU CROISEMENT DES SAVOIRS ET DES PRATIQUES, "Charte du croisement des savoirs et des pratiques avec des personnes en situation de pauvreté et d'exclusion sociale", http://www.atd-quartmonde.fr/IMG/pdf/Charte_du_Croisement_des_Savoirs_et _des_Pratiques.pdf (31 mars 2015).

14. Les Instituts de recherche en santé du Canada (IRSC) ont pour but à la fois de développer de nouvelles connaissances en recherche et de veiller à ce que ces nouvelles connaissances soient appliquées en vue d'obtenir des résultats pratiques. Les IRSC divisent l'application des connaissances en deux grandes 
deux chercheuses universitaires et deux volontaires permanentes du Mouvement ATD Quart Monde ont élaboré et réalisé en étroite collaboration la recherche ÉQUIsanTÉ.

ÉQUIsanTÉ est une recherche participative qui a permis un dialogue entre des cliniciens et cliniciennes et des personnes en situation de pauvreté afin d'identifier les barrières qui les séparent et de chercher des solutions pour améliorer la qualité et l'accès aux soins (voir encadré).

\section{Le but}

\section{La recherche participative ÉQUIsanTÉ ${ }^{15}$}

Améliorer la qualité et l'équité des soins offerts aux personnes en situation de pauvreté (PESP).

\section{Les objectifs}

1) Identifier les moyens et les actions nécessaires pour favoriser le déploiement de pratiques professionnelles orientées vers la compétence sociale au sein d'une clinique qui offre des soins primaires.

2) Identifier les facteurs favorisant l'implication des PESP dans le processus de développement de la compétence sociale.

\section{Les acteurs}

- 6 membres du Mouvement ATD Quart Monde, dont 4 vivant ou ayant vécu en situation de grande pauvreté et d'exclusion et 2 volontaires permanents.

- 15 membres d'équipes de soins de deux unités de médecine de famille de l'Université de Sherbrooke.

$$
\div+\div
$$

catégories : l'application des connaissances en fin de subvention (AC) et l'application des connaissances intégrée (ACI). Contrairement à l'AC, l'ACI consiste à engager et à intégrer ceux qui devront agir sur les résultats, les utilisateurs et utilisatrices des connaissances, dans le processus de recherche. S’inscrivant dans la démarche de recherche participative, l'ACI exige que les chercheurs et chercheuses ainsi que les utilisateurs et utilisatrices des connaissances forment des partenariats et s'engagent dans un processus de collaboration en se fixant comme impératifs la coproduction, l'échange et la mise en pratique des connaissances. Dans ce cadre, les IRSC ont produit un guide sur la collaboration entre ces personnes dans la recherche en santé, promouvant ainsi l'ACI comme un moyen efficace pour l'application des nouvelles connaissances. Voir David PARRY, Jon SALSBERG et Ann C. MACAULAY, Guide sur la collaboration entre chercheurs et utilisateurs des connaissances dans la recherche en santé, Groupe PRAM McGill, http://www.cihrirsc.gc.ca/f/documents/Guide_sur_la_collaboration_dans_la_recherche_en_sante.pdf (31 mars 2015).

15. Christine LOIGNON et al., "Perceived Barriers to Responsive Care for Persons Living in Poverty in Quebec, Canada. The EQUIhealhTY Project ", International Journal for Equity and Health, vol. 14, ${ }^{\circ} 4$, 2015, p. 1-11. 
- Un comité de pilotage ayant pour fonction de prendre des décisions concernant le contenu de la recherche (par exemple la formulation de la question de recherche), de prendre part à l'analyse des données, à l'interprétation des résultats et à la coécriture de documents d'analyse, et de participer à la diffusion des résultats : 2 chercheuses universitaires (dont une aussi professionnelle de la santé), 2 agentes de recherche, 2 volontaires permanents d'ATD Quart Monde, 2 personnes en situation de pauvreté et 1 professionnelle de la santé.

- Un comité de suivi ayant pour fonction de planifier le déroulement de la recherche, de décider des méthodologies utilisées, de participer à l'analyse et d'organiser la diffusion des résultats : 2 chercheuses, 2 agentes de recherche, 2 volontaires permanentes d'ATD Quart Monde. Ces personnes sont toutes aussi membres du comité de pilotage.

\section{Le déroulement}

- 19 rencontres du comité de pilotage (incluant des rencontres de travail en sous-groupes).

- 28 rencontres du comité de suivi.

- 3 rencontres de formation au photovoix ${ }^{16}, 3$ rencontres de photovoix entre groupes de pairs et 2 rencontres de croisement des savoirs de 3 heures entre les personnes en situation de pauvreté et les équipes de soins des deux unités de médecine familiale (clinique de médecine de famille) pour dialoguer à partir du travail du photovoix.

- Travail individuel, en groupes de pairs et en groupes mixtes tout au long du processus.

$$
\div+\div
$$

16. Le photovoix favorise la participation des personnes au processus de recherche et conduit, dans la plupart des cas, à des actions concrètes. Cette méthode de recherche participative, aussi appelée "photographie réflexive ", consiste à " utiliser la photographie pour représenter l'expérience et communiquer aux autres ses perceptions, émotions, et opinions qui parfois demeurent sous silence " (Ellen D. S. LOPEZ, Eugenia ENG, Naomi RoBINSON et Caroline C. WANG, «Photovoice as a Community-Based Participatory Research Method", Barbara A. ISRAEL, Eugenia ENG, Amy J. SchUlZ et Edith A. PARKER (dir.), Methods in Community-Based Participatory Research for Health, San Francisco, Jossey-Bass A Wiley Imprint, 2005, p. 326-348). 


\section{Les étapes}

- Formulation de la question de recherche en comité de pilotage: "Quelles sont les barrières entre les équipes de soins et les personnes en situation de pauvreté? "

- Répondre à la question de recherche, d'abord individuellement en prenant des photos, puis en groupes de pairs en utilisant la méthode du photovoix.

- Rencontre de croisement des savoirs entre les personnes en situation de pauvreté et les équipes de soins des deux unités de médecine de famille.

- Analyse des temps de croisement en groupes de pairs, puis par le comité de pilotage.

- Rédaction des résultats : une partie par le comité de pilotage, une autre partie par les agentes de recherche; coécriture de deux thèmes.

- Présentation des résultats dans les trois milieux respectifs (ATD Quart Monde et les deux unités de médecine de famille) par des membres du comité de pilotage.

- Élaboration des plans d'action dans les trois milieux.

- Temps de croisement et de questionnement mutuel autour des plans d'action.

- Mise en pratique des plans d'action dans les trois milieux.

- Évaluation de la mise en place des actions et des retombées anticipées.

À travers le compte rendu de cette recherche, nous illustrerons les conditions nécessaires à la mise en œuvre du croisement des savoirs avec des personnes en situation de grande pauvreté et d'exclusion sociale, telles qu'elles sont écrites dans la Charte du croisement des savoirs et des pratiques ${ }^{17}$.

$$
4+4
$$

17. Mouvement ATD QUART MONDE - ATELIERS DU CROISEMENT DES SAVOIRS ET DES PRATIQUES, "Charte du croisement des savoirs et des pratiques avec des personnes en situation de pauvreté et d'exclusion sociale»..., op. cit. 


\section{LES PRÉREQUIS ET LES CONDITIONS NÉCESSAIRES À LA MISE EN GEUVRE DU CROISEMENT DES SAVOIRS ET DES PRATIQUES}

Faire une recherche qui implique un croisement des savoirs suppose que chacun soit convaincu d'un changement nécessaire et ait la volonté d'y contribuer, mais aussi que chacun reconnaisse chez l'autre cette volonté de changement. Lors de désaccords, qui arrivent souvent dans un tel processus, il est important de se le rappeler.

Croiser les savoirs, c'est aussi considérer l'autre comme détenteur de savoirs, c'est être prêt à accueillir le savoir de l'autre et à accepter de remettre son propre savoir en question. C'est se placer ensemble dans une position de recherche, sachant que personne n'a la solution à lui seul.

Lors du projet ÉQUIsanTÉ, nous avons expérimenté ces conditions nécessaires indiquées dans la Charte du croisement des savoirs et des pratiques:

- La présence effective des personnes en situation de pauvreté dans toutes les étapes.

- Les conditions de l'autonomie des savoirs en vue de leur mise en réciprocité.

- L'établissement d'un espace de confiance et de sécurité afin de garantir les conditions de rigueur et d'échange.

- L'importance du rythme, de la durée et de l'accompagnement.

- La construction collective.

La présence effective des personnes en situation de pauvreté à toutes les étapes de la recherche

L'une des conditions pour réaliser le croisement des savoirs et des pratiques est que les personnes qui vivent en situation de pauvreté soient effectivement présentes tout au long du processus et non seulement à un moment précis pour donner leur témoignage sous forme d'exposé, de vidéo ou d'écrit.

Dans une philosophie similaire, Gélineau, Dufour et Bélisle ${ }^{18}$ proposent quatre constituantes nécessaires à toute recherche-action participative (RAP) : 1) dans une RAP, les cochercheurs et cochercheuses non universitaires prennent le droit de produire des connaissances scientifiques;

$$
4+4
$$

18. Lucie GÉLINEAU, Émilie DUfOUR et Micheline BÉLISLE, "Quand recherche-action participative et pratiques AVEC se conjuguent. Enjeux de définition et d'équilibre des savoirs ", Recherches qualitatives. Hors série, $\mathrm{n}^{\circ} 13,2012$, p. 35-54. 
2) une RAP vise à donner un droit de parole à des personnes qui vivent des situations d'exclusion, de subordination ou de marginalité ; 3) une RAP reconnaît un droit de penser, c'est-à-dire une capacité d'analyse critique, aux acteurs et actrices non universitaires; et 4) la RAP reconnaît aux cochercheurs et cochercheuses profanes le droit de cité, donc de propriété intellectuelle, sur les savoirs scientifiques qu'ils et elles ont contribué à produire, ainsi que le droit d'user de ces connaissances pour agir sur les rapports de pouvoir et contribuer au changement social dans une perspective de droit et de justice sociale.

Par ailleurs, les Instituts de recherche en santé du Canada (IRSC) expliquent ceci à propos de l'application des connaissances intégrée (ACI) :

Pour qu'il y ait une ACI, il faut au moins que les chercheurs et les utilisateurs des connaissances (1) décident ensemble de formuler les questions de la recherche, (2) interprètent les résultats de l'étude, (3) rédigent les messages au sujet des résultats et mettent les résultats de la recherche en pratique. Dans certaines situations, ce partenariat est élargi de manière à ce que les utilisateurs des connaissances soient aussi partenaires dans les décisions concernant la méthodologie de recherche, la mise au point des outils et la collecte des données ${ }^{19}$.

La présence effective des personnes en situation de pauvreté a été une réalité tout au long de la recherche ÉQUIsanTÉ, à travers la participation de deux personnes en situation de pauvreté au comité de pilotage. Ces deux personnes ont participé aux étapes de la formulation de la question de recherche, de la collecte des données, de l'analyse des données, de l'écriture des résultats ainsi que de la diffusion des résultats. Cela a demandé qu'on leur donne les outils et une formation, par exemple sur la codification et l'analyse de données, mais aussi que les universitaires acceptent de remettre en question et d'adapter leurs manières de travailler.

Une question importante est: qui sont les personnes en situation de pauvreté ayant la capacité et la légitimité de participer à une démarche de croisement des savoirs? Selon la charte, une des conditions à respecter est que ces personnes soient reliées à un groupe, qu'elles vivent l'association avec d'autres personnes en situation de pauvreté, qu'elles soient solidaires de leur milieu et ne l'aient pas quitté et qu'elles aient des espaces de réflexion et de dialogue en dehors de la recherche-action. Si une expérience personnelle

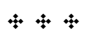

19. David PARry, Jon SALSBerg et Ann C. MaCAulay, Guide sur la collaboration..., op cit., p. 9. 
n'est pas reliée à un groupe social ou professionnel, elle reste fragile. C'est l'appartenance à un groupe social ou professionnel qui consolide le savoir dont une personne est porteuse. Voilà pourquoi ATD Quart Monde n'accepte pas que des personnes isolées participent à un croisement des savoirs ou à une recherche-action. Une personne ne peut s'improviser représentant du monde de la grande pauvreté.

Ainsi, les quatre personnes en situation de pauvreté qui ont participé à la recherche ÉQUIsanTÉ sont toutes des personnes membres de l'Université populaire Quart Monde ${ }^{20}$, un lieu de réflexion et de dialogue mis en place au Québec par le Mouvement ATD Quart Monde depuis 2007.

Les conditions de l'autonomie des savoirs en vue de leur mise en réciprocité Une autre condition pour la réalisation d'un croisement des savoirs est de garantir à tous et toutes la liberté de parole. Il est ainsi nécessaire que les cochercheurs et cochercheuses ne soient pas liés par des rapports de dépendance mutuelle.

Dans la recherche ÉQUIsanTÉ, cela voulait dire que les personnes en situation de pauvreté ne devaient pas être des patients d'un professionnel ou d'une professionnelle de la santé qui participait à la recherche.

Par ailleurs, puisque chaque personne engagée dans un croisement des savoirs a en référence son propre groupe d'appartenance ou de pairs au sein duquel elle trouve un espace de sécurité et de liberté pour bâtir sa propre pensée, la recherche ÉQUIsanTÉ a pris soin de prévoir des temps de travail en groupes de pairs. Par exemple, pour répondre à la question de recherche "Quelles sont les barrières entre les personnes en situation de pauvreté et les équipes de soins?", chaque personne a pris des photos (photovoix) qu'elle a d'abord présentées à ses pairs pour ensuite bâtir une réflexion commune à partager avec les autres groupes d'acteurs.

De la même façon, après l'écoute des réflexions d'un groupe de pairs, chaque groupe avait un temps pour lui permettre d'accueillir et de comprendre ensemble ce qui venait d'être dit et pour arriver à formuler une réflexion commune en réaction à ce qui venait d'être dit.

$$
\div \div
$$

20. Voir: MOUVEMENT INTERNATIONAL POUR L'ÉRADICATION DE LA PAUVRETÉ, «Rapport de l'évaluation participative sur l'Université populaire Quart Monde», http://www.atdquartmonde.ca/ rapport-de-levaluation-participative-sur-luniversite-populaire-quart-monde (31 mars 2015) et «Université populaire Quart Monde ", http://www.atdquartmonde.ca/nos-actions/universite-populaire-quartmondel (31 mars 2015). 


\section{L'établissement d'un espace de confiance et de sécurité afin de garantir les conditions d'échange et de rigueur}

Le croisement des savoirs et des pratiques n'est possible que si l'on assure un sentiment de sécurité et de confiance auprès de chaque personne par rapport à ses partenaires et au cadre instauré.

Pour la recherche ÉQUIsanTÉ, la préparation du formulaire de consentement, étape nécessaire de toute recherche réalisée avec des êtres humains, a été une expérience importante. D'abord, ce document a été préparé avec l'aide du comité de suivi, en vue de le soumettre au comité d'éthique de la recherche de l'université : il devait être le plus clair possible sur les objectifs et les modalités de la recherche. Ensuite, les volontaires permanents et les chercheurs et chercheuses ont pris le temps nécessaire pour s'assurer que les personnes en situation de pauvreté comprennent ce document sans s'en inquiéter. Si généralement la communauté scientifique considère le formulaire de consentement comme une protection pour les personnes qui participent à une recherche, signer un tel document peut être très inquiétant pour les personnes en situation de pauvreté à cause de leurs expériences négatives passées avec l'appareil bureaucratique, qui gère leurs droits, ou avec des marchands frauduleux.

Pour garantir les conditions d'échange, il ne suffit pas de se retrouver autour d'une même table. L'inégalité des positions est bien présente, car la reconnaissance sociale du savoir des uns et des autres n'est pas la même. Ce serait un piège de faire comme si tous les participants et participantes étaient d'emblée en situation d'égalité, alors que ce n'est pas le cas.

Dans le guide des IRSC sur l'application des connaissances intégrée, il est question de différences de pouvoir :

Les différences de pouvoir $-\mathrm{y}$ compris selon le genre/la race/l'âge/ l'éducation - peuvent empêcher certains utilisateurs des connaissances de dire exactement ce qu'ils pensent. Cette situation peut être particulièrement aiguë chez les partenaires communautaires (surtout ceux qui appartiennent à des communautés minoritaires ou marginalisées) qui peuvent se sentir intimidés à l'idée de travailler avec des gens appartenant au milieu universitaire. [...] Bien qu'il ne soit pas possible de faire disparaitre du jour au lendemain des différences de pouvoir bien ancrées, il est important d'en être conscient et de prendre les mesures nécessaires pour en atténuer l'effet sur la participation des utilisateurs des connaissances ${ }^{21}$.

$$
\div+\div
$$

21. David PArry, Jon Salsberg et Ann C. MacAulay, Guide sur la collaboration..., op. cit., p. 29. 
Rendre l'échange possible dans un tel contexte d'inégalités sociales est le rôle de l'équipe pédagogique, composée de personnes connaissant bien le milieu de la pauvreté pour l'avoir côtoyé depuis longtemps et de personnes connaissant bien le milieu professionnel ou universitaire. Ce rôle est de faire en sorte que tous puissent s'exprimer, mais en prêtant particulièrement attention aux personnes en situation de pauvreté.

Ainsi, les rencontres de croisement dans le cadre de la recherche ÉQUIsanTÉ ont toujours été coanimées par une volontaire permanente connaissant bien le milieu de la grande pauvreté et par une chercheuse universitaire connaissant bien le milieu professionnel de la santé. Toutes les rencontres de croisement ont été préparées de sorte que les personnes puissent y trouver leur place, sans se sentir bousculées ou sous pression, et se sentent à l'aise de contribuer par leurs savoirs.

Enfin, assurer l'atteinte d'une rigueur scientifique ou la conformité aux critères de qualité méthodologique représente des enjeux importants de la recherche-action participative, notamment pour faire reconnaître la valeur de ces travaux par la communauté scientifique et par les pouvoirs publics. C'est le rôle des universitaires d'y veiller et de donner aux cochercheurs et cochercheuses non universitaires les outils et les connaissances nécessaires pour qu'ils puissent jouer un rôle réel et actif dans toutes les étapes de la recherche ${ }^{22}$.

Dans le projet ÉQUIsanTÉ, le comité de suivi a pu bénéficier d'une première rencontre de formation offerte par le Participatory Research at McGill (PRAM) au début du projet, et un temps de formation de deux heures a été offert aux membres du comité de pilotage qui n'étaient pas des universitaires. Ces formations avaient pour but de donner à chacun une connaissance des méthodes de recherche et d'analyse à utiliser dans les étapes subséquentes du projet et ainsi de favoriser la participation de toutes les personnes du comité de pilotage, peu importe leur niveau de formation ou leur expérience antérieure en recherche.

\section{L'importance du rythme, de la durée et de l'accompagnement}

Croiser les savoirs demande de respecter le rythme de chacun, c'est-à-dire le rythme de compréhension et le rythme d'expression. Croiser les savoirs, c'est adapter une pédagogie du plus lent, parce que nous sommes convaincus que la

$$
\div \div
$$

22. Lucie GÉlineau, Émilie Dufour et Micheline BÉLISLE, "Quand recherche-action participative et pratiques AVEC se conjuguent. Enjeux de définition et d'équilibre des savoirs »...op. cit., p. 35-54. 
personne la plus lente a des choses à nous apprendre et que, grâce à elle, nous irons plus loin, au profit de tous. Croiser les savoirs ne se fait pas vite, cela prend du temps, c'est quelque chose qui se construit dans la durée, car cela demande maturation.

Dans le cadre de la recherche ÉQUIsanTÉ, après une année de mise en œuvre, le comité de suivi a compris que, pour permettre la participation effective des personnes en situation de pauvreté dans le processus de recherche, il fallait accepter d'avoir des réunions de comité de suivi plus fréquentes et des réunions de comité de pilotage moins chargées, ou d'avoir des périodes réservées aux groupes de pairs durant les rencontres du comité de pilotage. Il a fallu s'ajuster en cours de route.

Souvent, le temps, ou plutôt le manque de temps, est un piège qui fait en sorte que l'on retombe dans les vieilles habitudes: on fait les choses tout seul, car cela va plus vite. Ne pas tomber dans ce piège demande une vigilance continue. Aussi, le calendrier, les enjeux et les réalités des universitaires ne permettent pas toujours de respecter les exigences d'une recherche en croisement des savoirs, ce qui peut engendrer des malentendus, voire des " conflits ». Prendre le temps nécessaire pour comprendre ces malentendus et ces conflits, comprendre la réalité et les enjeux des uns et des autres et bâtir des accords font partie d'une recherche en croisement des savoirs.

Dans le projet ÉQUIsanTÉ, le comité de suivi a eu recours à une consultante externe, experte de la recherche participative avec des personnes marginalisées, pour aider à dénouer des incompréhensions mutuelles. La consultante, choisie d'un commun accord, a d'abord dialogué avec les membres par groupes de pairs (chercheurs universitaires et non universitaires) afin de faciliter une rencontre de mise en commun. Elle a aidé le comité de suivi à identifier des pistes de compromis adaptées à la réalité d'une recherche participative.

Permettre à des personnes en situation de pauvreté d'aller au bout de la recherche, et d'y apporter une vraie contribution, demande un accompagnement dans la durée par des personnes qui connaissent bien le milieu de la pauvreté. Accompagner les personnes en situation de pauvreté dans un projet de recherche, c'est les aider à s'exprimer avec leurs propres mots et ne jamais se substituer à elles; leur permettre de construire une pensée commune avec leurs pairs ; les soutenir dans la compréhension des autres acteurs; les accompagner avant et après les rencontres. Il s'agit du rôle de l'organisme communautaire ou de l'association citoyenne qui s'engage dans la recherche. Il est important de prévoir dans le budget une compensation financière pour ces organismes communautaires. 
Dans le cadre de la recherche ÉQUIsanTÉ, c'est une volontaire permanente d'ATD Quart Monde qui a réalisé ce mandat tout au long du processus. Par exemple, avant chaque comité de pilotage, de préférence le jour précédent, cette volontaire réunissait les deux personnes en situation de pauvreté pour que celles-ci puissent lire les documents, comprendre l'ordre du jour et préparer leurs contributions pour la réunion. Notons qu'à chacune des rencontres, les personnes en situation de pauvreté ont reçu une compensation financière pour leur travail, signe de reconnaissance de leur savoir et du travail fourni.

\section{La construction collective}

Le meilleur moyen de confronter réellement les points de vue est de s'engager mutuellement, lorsque c'est possible, dans une production commune.

Croiser les savoirs nécessite d'accepter la confrontation, car, souvent, il n'y a pas de construction collective possible sans confrontation. Identifier les éléments de désaccord est une étape essentielle avant de construire ensemble. Faire ce travail avec des personnes en situation de pauvreté ne s'improvise pas: cela requiert du temps et l'adoption d'une méthodologie adaptée.

Voici, à titre d'illustration, les étapes qui ont été suivies pour faire les analyses croisées et une écriture collective lors de la recherche ÉQUIsanTÉ:

- Travail individuel: chaque membre du comité de pilotage lisait les extraits de transcriptions attribués à un thème et soulignait quatre phrases qui lui semblaient très importantes: deux phrases dites par des personnes en situation de pauvreté et deux phrases dites par des professionnels de la santé.

- Travail en groupe de pairs: les personnes en situation de pauvreté se rencontraient ensuite pour se présenter leur travail et bâtir une réflexion commune.

- Travail en croisement lors des rencontres du comité de pilotage: au début des réunions, quelques minutes étaient prises pour que chacun puisse librement exprimer ses premières réactions, son ressenti ou ses incompréhensions à la lecture des transcriptions qui portaient sur le thème retenu. Par la suite, chaque personne partageait les phrases qu'elle avait trouvées importantes lors de la lecture des transcriptions en expliquant son choix. Puis, une séance de travail en groupes de pairs avait lieu à la mi-temps de la rencontre afin de travailler sur un plan 
résumant l'essentiel de ce qui avait été dit sur le thème. Après ce temps de travail en pairs, les groupes présentaient leur document aux autres membres du comité de pilotage et un plan final, contenant des éléments de chacun, était adopté par l'ensemble du groupe.

À la suite de ces rencontres d'analyse, deux ou trois membres du comité de pilotage (universitaire/non universitaire/personne en situation de pauvreté) se proposaient pour travailler à la rédaction d'un texte ou d'un paragraphe du texte. Une rotation a été faite entre les différentes séances de travail afin que plusieurs personnes puissent participer au processus d'écriture. Une fois terminé, le texte était envoyé aux autres membres du comité de pilotage et une rencontre avait lieu afin que chacun partage ses réactions, ses suggestions et ses commentaires. Lors de cette rencontre, le texte était modifié et ajusté lorsqu'il y avait un consensus sur les changements à apporter. Ce processus a été répété à plusieurs reprises, car un sous-groupe (jamais une personne seule) retravaillait le texte à la suite des modifications suggérées en comité de pilotage. Lors de la dernière réunion, les textes ont été relus et chaque personne a pu proposer des dernières modifications. Les textes ont donc été modifiés jusqu'à ce qu'ils soient satisfaisants pour l'ensemble du groupe.

L'analyse de deux thèmes choisis par les membres du comité de pilotage, incluant les personnes en situation de pauvreté, a donc été entièrement participative, car chaque membre du comité de pilotage a activement été impliqué dans le travail, du début à la fin, pour identifier et décrire les éléments importants associés aux thèmes, interpréter ces données et rédiger les textes. Ce travail a nécessité six rencontres du comité de pilotage et sept rencontres en sous-groupes, en plus des rencontres de préparation des membres d'ATD Quart Monde aux rencontres du comité de pilotage et aux séances de travail en sous-groupes.

\section{DE NOUVEAUX CONTENUS DE SAVOIR, UNE COMPRÉHENSION PLUS FINE DE LA RÉALITÉ ET DE NOUVEAUX LIENS SOCIAUX}

La démarche du croisement des savoirs vise à produire de nouveaux contenus de savoir et une compréhension plus fine de la réalité. Par exemple, la recherche ÉQUIsanTÉ a permis de découvrir combien les logiques des uns et des autres, et surtout la non-compréhension de ces logiques, sont une barrière entre les personnes en situation de pauvreté et les équipes de soins: 
Lors d'une des rencontres de croisement des savoirs, des discussions ont eu lieu sur le choix qu'ont les mamans qui viennent d'accoucher: allaiter leur enfant ou lui donner du lait maternisé. C'est un exemple flagrant qui illustre bien comment deux logiques peuvent s'opposer. D'une part, pour le professionnel de la santé, c'est le lait maternel qui est le meilleur choix : il est très bon pour la santé du bébé et plus économique pour le budget familial. [...] Et d'autre part, une personne en situation de pauvreté expliquait que le choix du lait maternisé peut venir de la crainte de ne pas pouvoir donner au bébé tout ce dont il a besoin dans son lait maternel. La maman peut aussi croire que son lait ne sera pas bon pour bien nourrir son enfant, parce qu'on lui a souvent dit "Tu n'es pas bonne», "tu ne vas pas réussir», donc elle se dévalorise. [...] En conséquence, chacun fait des choix différents et a des priorités différentes, ce qui amène de l'incompréhension et du jugement de part et d'autre si on ne comprend pas la logique de l'autre comme l'a exprimé [cette professionnelle] : Ça, j'aurais jamais pu le savoir dans le bureau. J'avais demandé à la femme, « est-ce que vous allaitez, ou vous donnez du lait maternisé ?"

Elle me dit, «je donne du lait maternisé ». Je suis pas sûre que je lui aurais demandé pourquoi, puis je suis pas sûre que ç'aurait ressorti aussi, «bien, je donne du lait maternisé parce que j'ai peur que mon enfant ait pas tout ce qu'il lui faut dans mon lait, vu que je mange pas assez ». Peut-être que la personne me l'aurait jamais dit, qu'elle mangeait juste une fois par jour, puis que ça l'inquiétait pour son enfant. Ça fait que je vais sortir en me disant, elle prend du lait maternisé, ça va lui coûter super cher, c'est pas un bon choix. Puis elle, elle a l'impression que je la comprends pas ${ }^{23}$.

Mais la démarche de coconstruction de savoirs est aussi une démarche de création continue de nouveaux liens sociaux. En valorisant des savoirs jusquelà méconnus, ce sont les personnes chez qui on reconnaît ces savoirs que l'on valorise. Non seulement cette démarche crée la communication entre des milieux qui ne se connaissent pas, mais elle provoque chez les participants et participantes des prises de conscience transformatrices qui les amènent à la fois à reconstruire leur propre savoir (de soi, des autres et des choses), leur mode de relation aux autres, et à changer leurs pratiques. Une professionnelle, participante de la recherche ÉQUIsanTÉ, exprimait ainsi sa prise de conscience concernant la logique du diagnostic:

$$
4+4
$$

23. Christine LOIGNON et al., Projet ÉQUIsanTÉ. Rapport de recherche, p. 18-19, https://www.atdquartmonde.fr/projet-equisante-rapport-de-recherche/ (31 mars 2015). 
Moi je fais référence à mon expérience personnelle, souvent un dossier d'hospitalisation, le patient qui est hospitalisé une semaine, exemple on remarque qu'il est un peu lent ou il comprend pas bien, en quelque part quelqu'un va écrire... retard intellectuel, point d'interrogation là. Finalement dans le résumé, ça va être écrit retard intellectuel, là finalement d'hospitalisation en hospitalisation, de consultation en consultation, ce diagnostic-là se promène, finalement la personne est étiquetée et même les gens vont moduler leurs interventions en fonction de ce diagnostic-là qui oui ou non est vrai ou pas vrai, mais est-ce que ça sert à quelque chose de l'écrire? [...] moi en tout cas ça me fait réfléchir ${ }^{24}$.

Ou encore, une personne en situation de pauvreté qui a pris conscience de la façon dont elle percevait son médecin et dont elle interagissait avec lui :

Bien en tout cas moi je vais voir mon médecin de façon un peu différente et je vais arrêter de le chicaner. [...] Je vais le voir comme une personne et non pas comme un professionnel qui a toutes les connaissances et qui peut tout... ${ }^{25}$

Ainsi la démarche de croisement est-elle aussi importante que les nouveaux contenus de savoir qu'elle vise à produire. C'est une démarche de coformation réciproque où chacun est cochercheur, coacteur et coformateur.

\section{CONCLUSION}

Faire une recherche en croisement des savoirs n'est pas un chemin sans écueils, mais la recherche ÉQUIsanTÉ a montré que cela améliore notre compréhension des mécanismes de la pauvreté et de l'exclusion. En ce sens, la démarche du croisement des savoirs et des pratiques n'est pas une simple démarche de participation des populations en situation de pauvreté, ni même la mise en œuvre d'une démarche permanente de démocratie participative au sein de laquelle les personnes en situation de pauvreté sont actrices à part entière. C'est aussi une source précieuse de savoirs, un espace collaboratif de construction de connaissances qui ne pourraient pas être obtenues autrement et qui viennent nourrir de manière fine la réflexion sur les inégalités sociales et les politiques publiques qui veulent les réduire. Cette démarche montre aussi qu'il est possible et pensable que la recherche scientifique ne laisse personne de côté et fasse participer des personnes de tout horizon, que nos sociétés deviennent de véritables sociétés du savoir, sans exclusion.

$$
+4
$$

24. Ibid., p. 21.

25. Ibid., p. 33.

LE CROISEMENT DES SAVOIRS ET DES PRATIQUES AVEC DES PERSONNES EN SITUATION DE PAUVRETÉ 
La finalité de la démarche du croisement des savoirs avec des personnes en situation de pauvreté et d'exclusion sociale est bien un changement social en vue d'une plus grande justice, par la médiation des savoirs des uns et des autres. 